\title{
Etude de la dynamique des populations d'Aedes echinus (Culicide dendrolimnique) en écophase aquatique, au Maroc
}

\author{
G. Metge1 \\ M. Elalaoui1
}

Mots clés: Diptera, Culicide dendrolimnique, Maroc, dynamique des populations, autorégulation.

Le développement et les variations quantitatives des populations d'Aedes echinus dépendent du volume des précipitations, de la température, du parasitisme et du cannibalisme. Ce phénomène d'autorégulation favorise la nymphose en diminuant la densité larvaire et en suppléant les carences protéiniques et lipidiques d'un milieu pauvre.

A study of the population dynamics of the aquatic stages of Aedes echimus (dendrolimnic Culicidae) in Morocco.

Keywords : Diptera, dendrolimnic Culicidae, Morocco, population dynamic.

The development and quantitative changes in the populations of Aedes echinus depended upon the amount of precipitation, temperature, parasitism and cannibalism. The phenomenon of « autoregulation » favours pupation by reducing larval density and by supplementing the protein and lipid deficiences of the poor environment.

\section{Introduction}

Les suberaies de Ben Slimane et Beni Abid (cf. fig. 1), situées au coeur de la Méséta occidentale marocaine (pays Zaer) se composent de chênes lièges dont certains individus offrent à leur base des creux susceptibles de retenir l'eau des précipitations.

Ces cavités définissent un habitat particulier aux populations d'Aedes echinus : ElAlaoui (1985), Belakoul (1985), Benjaber (1985), Hajbaoui (1985).

Compte tenu de l'agressivité de ce Culicide sur les populations locales, il nous a semblé intéres. sant d'étudier cette espèce d'une manière quantitative. L'absence de marqueur radioactif et la difficulté à comptabiliser les œufs nous ont conduit à analyser l'écophase aquatique, en tenant compte des abondances cumulées des différentes générations.

1. Laboratoire d'écologie A.I.44, Ecole Nortnale Supérieure, Mers Sultan B.P. 9172 Casablanca, Maroc.

\section{Dénombrement des stades larvaires}

L'eau du creux, de 2 stations (Ben Slimane et Beni Abid) vidée régulièrement (une fois par semaine du $1 / 12 / 83$ au 20/03/84) dans un filet langeron à l'aide de petits récipients en plastique, est recueuillie dans un seau gradué permettant d'apprécier directement le volume.

Le contenu du filet représente la faune plus des débris de feuilles mortes et des impuretés que l'on transvase dans une bassine blanche remplie de l'eau du gîte, on procède au tri et comptage des différents stades larvaires. La récolte des larves enfouies dans la vase nécessite le rinçage du creux par le même procédé.

\section{Détermination des âges}

Les mesures portent principalement sur les exuvies correspondantes à chaque stade larvaire. Ces données statistiques nous ont permis de définir des 


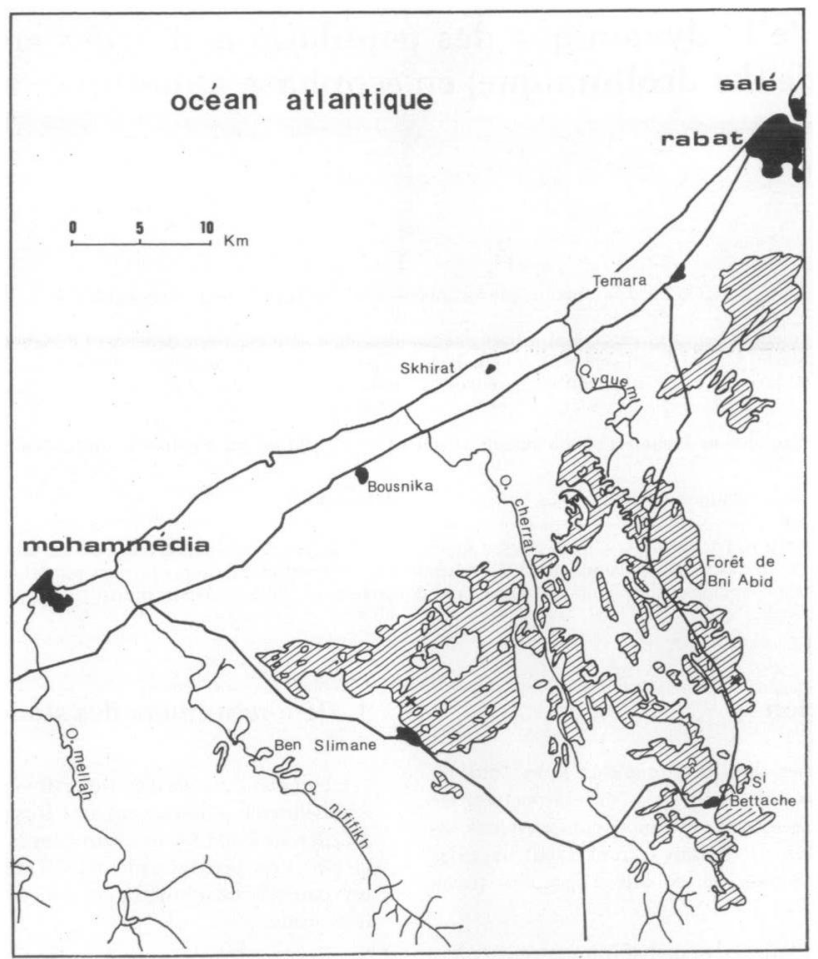

Fig. 1 : Localisation géographique des stations étudiées.

classes d'âge repérables sur le terrain à l'aide d'une loupe binoculaire équipée d'un micromètre.

\subsection{Les histogrammes de taille (fig. 2)}

A partir d'une étude effectuée au laboratoire sur un échantillon considérable d'Aedes echinus, prélevé sur plusieurs gites des stations de Ben Slimane, Sidi Bettache, nous obtenons les résultats consignés sur le tableau 1.

\subsection{Analyse des histogrammes de taille}

Lorsque la période de reproduction est nettement saisonnière, la structure par tailles des populations peut faire apparaître des modes bien distincts, correspondant à autant de classes d'âge successives. Dans notre cas les modes successifs ne sont pas discernables.

Différentes méthodes permettent, au prix de certaines hypothèses, d'analyser les histogrammes de 


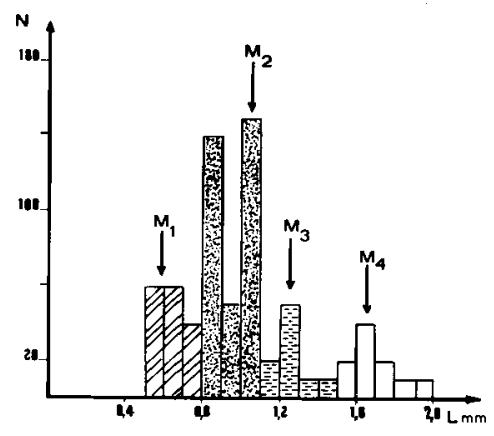

Ben Slimane

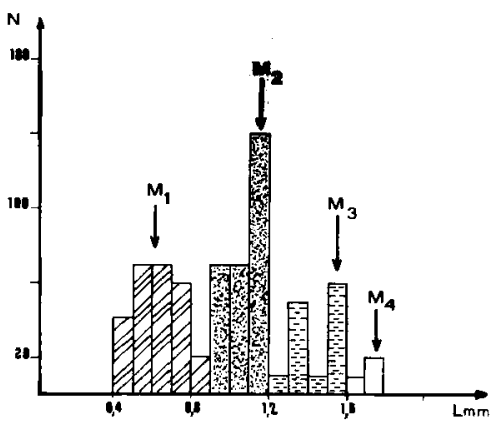

Sidi Bettache

Fig. 2 : Détermination statistique de l'âge d'Aedes echinus

taille. Si l'on admet que pour chaque cohorte d'âge déterminé, il existe une distribution symétrique des tailles autour du mode, on dégagera des modes " masqués" par la méthode des maximum successifs. Ces valeurs de taille donnent des indications moyennes pour A. echinus. Or, il convient de tenir compte des divers paramètres tels que l'influence de la nourriture, la densité, l'exposition etc... pouvant entraîner des variations. Pour diminuer les
Tableau I : Etude biométrique des larves d'Aedes echinus SIDI BETTACHE BEN SLIMANE

\begin{tabular}{ccc}
\hline $\mathbf{L}(\mathrm{mm})$ & Effectif & Effectif \\
\hline 0,4 & $\mathbf{4 0}$ & $\mathbf{0}$ \\
0,5 & 0 & $\mathbf{6 0}$ \\
0,6 & 60 & $\mathbf{6 0}$ \\
0,62 & 10 & $\mathbf{0}$ \\
0,7 & 70 & $\mathbf{4 0}$ \\
0,8 & 60 & $\mathbf{1 4 0}$ \\
0,9 & 20 & $\mathbf{5 0}$ \\
1 & 70 & $\mathbf{1 5 0}$ \\
1,1 & 10 & $\mathbf{2 0}$ \\
1,2 & 140 & $\mathbf{5 0}$ \\
1,3 & 20 & $\mathbf{1 0}$ \\
1,4 & 50 & $\mathbf{1 0}$ \\
1,5 & 10 & $\mathbf{0}$ \\
1,6 & 60 & $\mathbf{4 0}$ \\
1,7 & 10 & $\mathbf{0}$ \\
1,8 & 20 & $\mathbf{2 0}$ \\
1,9 & 0 & 0 \\
2 & 0 & 10
\end{tabular}

risques d'erreurs, nous avons déterminé les classes de taille pour chacune des stations étudiées.

\section{Les variations d'abondance dans le temps : interprétation des résultats}

L'analyse des histogrammes d'abondance (fig. $4,5, c)$ soulève deux problèmes :

- les fortes variations des effectifs au cours du temps,

- la "stase " enregistrée d'une manière génèrale au niveau des quat rièmes stades.

Pour résoudre ces problèmes il convient de corréler les observations et les mesures faites sur le terrain et (ou) en laboratoire.

\subsection{Influence des facteurs ablotiques}

L'eau, principal facteur actif, agit de deux façons :

- par le volume qu'elle occupe dans le creux d'arbre,

- par sa qualité.

- Le volume de l'eau est déterminé par l'abondance et la fréquence des précipitations au cours de l'année (Fig. 3). En superposant ces données météorologiques aux histogrammes d'abondance, on 


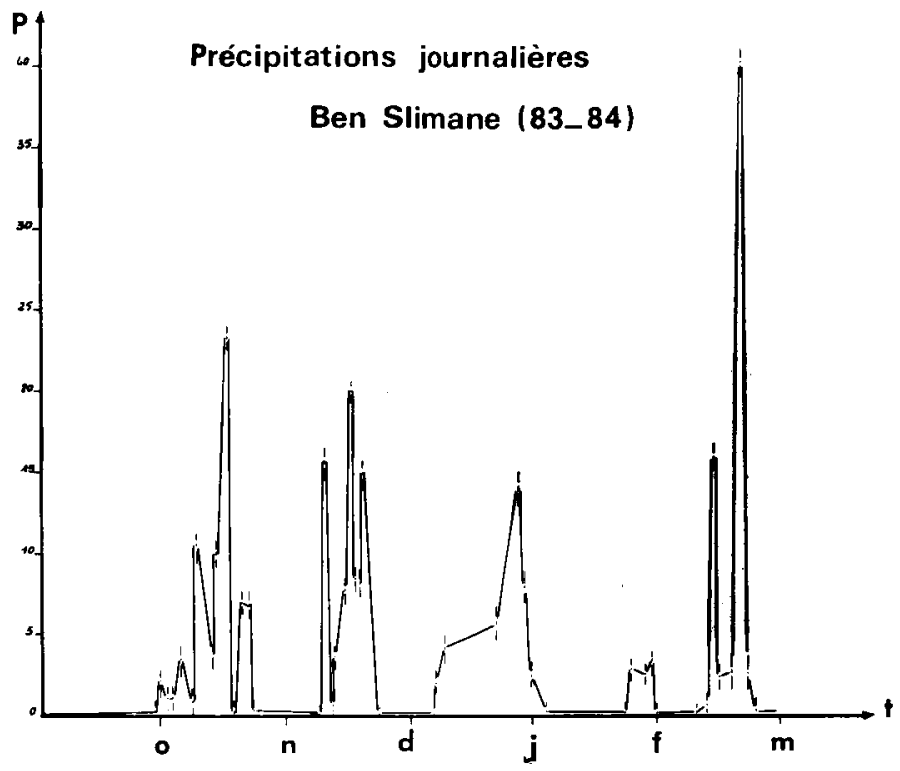

Fig. 3 : Précipitations journalières, Ben Slimane 1983-1984.

constate l'apparition des larves $L_{1}$ après chaque pluie importante ; ces poussées successives correspondent à autant d'éclosions et démontrent l'existence de 3 générations au niveau des 2 stations.

Les pontes se réalisent à des niveaux différents dans les creux et les cufs se présentent sous forme de stocks étagés sur les parois. Les variations du volume d'eau déterminent des niveaux privilégiés permettant l'éclosion lorsque la submersion se conjugue à des conditions physico-chimiques favorables (température, phénomènes de réduction etc...). Les précipitations importantes peuvent jouer un rôle négatif en provoquant le débordement et la disparition d'un grand nombre de larves.

\section{- Qualité de l'eau}

La constance de la qualité physico-chimique des eaux: pH, conductivité, dureté calcique etc...
(Rioux 1958. FlAlaoui 1985) n'apporte pas de résultats significatifs permettant des corrélations avec les effectifs larvaires ou leur développement.

La température, seule, présente un certain intérêt.

Le développement larvaire et les métamorphoses se produisent lorsque la température de l'eau s'élève. Toutefois, son action reste secondaire au niveau de la nymphose : bien que le seuil thermique de $12^{\circ} \mathrm{C}$ soit nécessaire (fig. 4 et 5 ) l'intervention de ce facteur seul n'est pas suffisante. Il faut donc envisager l'action combinée d'un ou plusieurs autres facteurs.

\subsection{Influence des facteurs biotiques}

- Les interrelations de la communauté dendrolimnique

D'après nos observations le phénomène de prédation par les Tanypodinés (Chironomides) et autres 

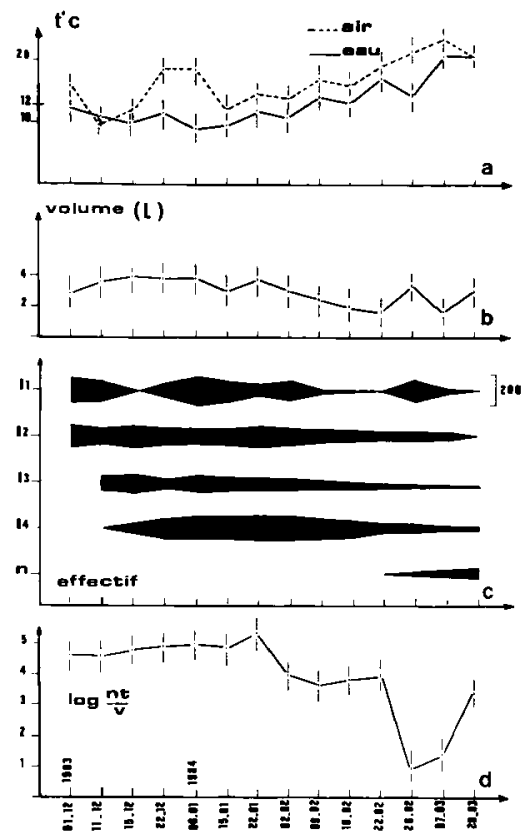

Fig. 4 (a), (b), (c), (d) : Variation des effectifs en fonction de la température et du volume d'eau: Ben Slimane.

Diptères (Chaoborinés, Culicinés) ne semble pas important, leur densité ne dépasse jamais quelques individus.

La mortalité, enregistrée sur les populations d'Aedes echinus, affecte surtout les stades les plus jeunes, elle dépend pour une grande part du parasitisme par les Leptothrix et autres bacilles.

La faible densité des Culicides associés : Aedes geniculatus, A. berlandi, A. pulchritarsis ne semble pas favorable à une compétition interspécifique. D'autant que ces espèces se relaient dans la colonisation de l'habitat.
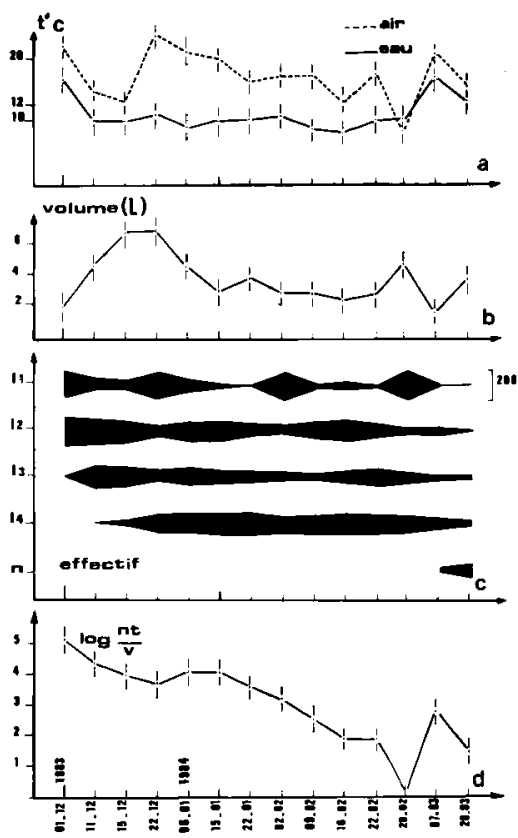

Fig. 5 (a), (b), (c), (d) : Variation des effect if s en fonction de la température et du volume d'eau : Sidi Bettache.

\section{- La régulation de la population}

Si une véritable compétition interspécifique n'apparaît pas évidente, par contre un phénomène d'autorégulation de la population paraît plus marqué.

\section{a) La densité}

Les travaux de Moore \& Fisher (1969), Moore \& Whitacre (1972), Whittaker \& Feeny (1971) montrent que les larves de moustiques libèrent une substance appelée G.R.F. (Growth Retardant Factor) qui retarde le développement. La production de cette substance est d'autant plus grande que la densité larvaire est élevée et que la quantité de nourriture est faible. En observant les histogrammes ( $\mathrm{fg} .4 \mathrm{c}$ et 5c) on se rend compte que la nymphose se déclenche lorsque la densité de l'effectif général diminue et la température de l'eau s'élève. 
Nous avons étudié ces trois paramètres dans le temps en utilisant pour chaque observation la formule suivante $: R=\log \left(\frac{\mathrm{Nt}}{\mathrm{V}}\right)$.

Nt étant le nombre d'individus d'Aedes echinus, $V$ le volume de l'eau du gite.

Les résultats consignés sur les figures 4 et 5 (d), mettent en évidence des corrélations entre:

- la température (seuil thermique de $12^{\circ} \mathrm{C}$ )

- le rapport $\mathbf{R}$ (valeurs inférieures à 3,5 ) et le déclenchement de la nymphose.

\section{b) Le cannibalisme}

La diminution de la densité, importante dans le développement de la population, peut être liée : au parasitisme des premiers stades, aux fortes pluies (débordement). L'évaluation quantitative de ces phénomènes ( 5 à $10 \%$ ) démont re que la chute de l'effectif ne peut leur être totalement imputable.

Dans des conditions expérimentales El Alaoui (1985) a démontré l'action du cannibalisme des larves de $4^{e}$ stade sur des larves de stade 1 et 2 , lorsque la température de l'eau est supérieure à $12^{\circ} \mathrm{C}$; cet apport protéinique déclenche la nymphose.

Dans le milieu naturel, en absence de photosynthèse, seules les bactéries constituent le régime alimentaire d'Aedes echinus. Or la nymphose nécessite une consommation d'énergie élevée (Chauvin 1956, Raccaud-Schœller 1980), qui se réalise normalement à partir de substances lipidiques accumulées dans la phase préimaginale. La consommation sélective des jeunes stades va:

- combler les carences alimentaires subies par les larves L4

- diminuer la densité et le G.R.F.

\section{Conclusion}

L'étude dynamique des populations d'Aedes echinus en écophase aquatique nous a permis de mettre en relief les causes des fluctuations de l'effectif au niveau des stations retenues.

Ces variations d'abondance sont déterminées par :

- les facteurs exogènes (précipitations) et endogènes (facteurs d'éclosion) responsables de l'apparition des différentes générations ;

- la mortalité liée essentiellement au parasitisme et au cannibalisme.
Nous avons pu mettre en évidence le rôle du cannibalisme comme facteur de régulation de la population et son action déterminante au niveau du déclenchement de la nymphose, après une stasc de plusieurs mois des larves du quatrième stade.

C'est l'action simultanée du seuil thermique de $12^{\circ} \mathrm{C}$, de la consommation des jeunes larves par des individus du quatrième stade, des faibles densités qui conditionnent la nymphose.

Travaux cttés

Belakoul (N.). 1985. - Contribution à l'etude de la faune culicidienne dendrolimnique de la suberaie en Pays Zaer (Maroc). Colonisation par Culex pipiens d'un nouvel habitat : causalité et étude comparée de deux formes de Culex pipiens. D.E.A. Universite $P$. Sabatier Toulouse: $113 \mathrm{p}$.

Benjaber (M.). 1985. - Contribution à l'étude de la faune culicidienne dendrolimnique de la suberaie en Pays Zaer (Maroc). Aspect systématique. D.E.A. Université Paul Sabatier Toulouse: $111 \mathrm{p}$.

Chauvin (R.). 1956. - Physiologie de I'Insecte. I.N.R.A. Paris : 916 p.

Elalaoui (M.). 1985. - Contribution à l'étude de la faune culicidieme dendrolimmique de la suberaie en Pays Zaer (Maroc). Aspect écophysiologique. D.E.A. Université P. Sabatier Toulouse : $86 \mathrm{p}$.

Hajbaoui (S.). 1985. - Contribution à l'étude de la faune culicjdienne dendrolimnique de la suberaie en Pays Zaer (Maroc). Cartographie écologique. D.E.A. Université P. Sabatier Tou. louse : $94 \mathrm{p}$.

Moore (C.G.) \& Fisher (B.R.). 1969, - Competition in mosquitces. Density and species ratio effects on growth, mortality, fecundity, and production of growth retardant. Ann. ent. Soc. Am. $62,6: 1325 \cdot 1331$.

Moore (C.G.) \& Whitagre (D.M.). 1972. - Competition in mosquitoes. 2. Production of Aedes aegypti larval growth retardant at various densities and nutrition levels. Ann. ent. Soc. Am., $65,4: 915-918$.

Raccaud-Schoeller (J.). 1980. - Les insectes, physiologie, développement. Masson (éd.), Paris : $296 \mathrm{p}$.

Rioux (J.A.). 1958. - Les Culicides du Midi oméditerranéen. Enc. ent. XXX. P. Lechevalier (éd.), Paris : 303 p.

Seguy (E.) 1950. - La biologie des Dipteres. Enc. ent., A. XXVI, P. Lechevalier (éd.), Paris : 607 p.

Whit taker (R.H.) \& Feeny (P.P.). 1971. - Allelochemics, chemical interactions between species. Science, N.Y., $171: 757-770$. 